\title{
Note sur des structures mégalithiques en Guyane brésilienne, Amapá
}

\section{Mariana Petry Cabral et João Darcy de Moura Saldanha}

Traducteur : l'anglais par Stéphen Rostain

\section{(2) OpenEdition Journals}

Édition électronique

URL : https://journals.openedition.org/jsa/10803

DOI : 10.4000/jsa. 10803

ISSN : $1957-7842$

Éditeur

Société des américanistes

Édition imprimée

Date de publication : 23 juillet 2009

Pagination : $97-100$

ISSN : 0037-9174

Référence électronique

Mariana Petry Cabral et João Darcy de Moura Saldanha, « Note sur des structures mégalithiques en Guyane brésilienne, Amapá », Journal de la Société des américanistes [En ligne], 95-1 | 2009, mis en ligne le 10 juin 2014, consulté le 04 septembre 2022. URL : http://journals.openedition.org/jsa/10803 ; DOI : https://doi.org/10.4000/jsa.10803 


\title{
NOTE DE RECHERCHE
}

\section{NOTE SUR DES STRUCTURES MÉGALITHIQUES \\ EN GUYANE BRÉSILIENNE, AMAPÁ}

\author{
Mariana PETRY CABRAL et João DARCY DE MOURA SALDANHA *
}

\section{INTRODUCTION}

L'archéologie de l'État d'Amapá a une longue histoire, qui commence à la fin du XIX ${ }^{e}$ siècle avec les études de Ferreira Penna (1879) et d'Emilio Goeldi (1905). Néanmoins, peu de projets de fouilles systématiques ont été menés dans cette région, d'où la rareté des informations sur la diversité et le détail des caractéristiques de l'héritage archéologique. Bien que l'on connaisse plusieurs sites dans l'ensemble de l'État et au moins trois phases archéologiques définies de façon historico-culturelle (phases Mazagão, Aristé et Aruã ; Meggers et Evans 1957), jusqu'au début de notre projet dans l'Amapá septentrional, il n'existait pas d'informations précises sur les gisements à structures mégalithiques.

La prospection que nous avons menée dans cette zone a fourni de nouvelles données archéologiques, indiquant une présence bien plus importante que prévue de structures mégalithiques et d'autres types de sites. Avant de commencer notre projet, dix sites mégalithiques avaient été répertoriés, localisés principalement entre les fleuves Cunani et Flechal sur le littoral nord d'Amapá. Grâce à nos travaux, dix autres ont pu être identifiés en plus d'autres types de sites, tels des concentrations céramiques de plein air, des grottes funéraires et des dépôts céramiques dans des endroits spéciaux. À ce jour, 53 sites sont connus dans toute la zone étudiée.

* IEPA/Gerência de Pesquisa Arqueológica, Instituto de Pesquisas Científicas e Tecnológicas do Estado do Amapá, av. Feliciano Coelho, 1509, CEP 68901-025, Macapá, Amapá, Brasil [mariana.cabral@iepa.ap.gov.br] [joao.saldanha@iepa.ap.gov.br].

Journal de la Société des Américanistes, 2009, 95-1, pp. 97-110. OSociété des Américanistes. 
L'enregistrement précis réalisé des structures mégalithiques montre une grande diversité de formes et de dimensions, autant qu'une grande proximité entre certaines d'entre elles. Les sites mégalithiques sont, pour la plupart, organisés selon une distribution circulaire de groupes de blocs de granite en position horizontale, verticale ou oblique, localisés au sommet de collines. Les dimensions et les compositions sont variables. Certains sont plutôt petits, avec moins de $10 \mathrm{~m}$ de diamètre, et formés de blocs mesurant moins d'un mètre de longueur. La plus grande structure circulaire découverte (AP-CA-18 ${ }^{1}$ ) mesure plus de $30 \mathrm{~m}$ de diamètre et est constituée de grands blocs, dont certains s'élevant à plus de $3 \mathrm{~m}$ au-dessus du sol. Quelques structures mégalithiques présentent des plans irréguliers ou linéaires, avec des blocs disposés parallèlement aux rivières. Dans ces cas, les collines sur lesquelles les sites sont installés sont relativement étroites, réduisant l'espace disponible pour installer les ensembles, qui peuvent mesurer jusqu'à plus de $100 \mathrm{~m}$ de longueur (Nimuendajú 2004) et $5 \mathrm{~m}$ de largeur.

Pour dresser une carte archéologique, il est essentiel de s'intéresser à tous les types de gisements qui ont été trouvés. Parmi les 53 sites archéologiques identifiés, 20 possèdent des structures mégalithiques, 8 sont des grottes ou des abris sous roches funéraires, 4 sont des sites à polissoirs et les 21 derniers sont différents types de concentrations de céramiques. Parmi ces derniers sites, il y a des caches évidentes - ainsi, un agencement de poteries sous l'eau (un site) ${ }^{2}$, un dépôt de poterie au sommet du substrat rocheux (un site) et, au moins, deux sortes de dépôts funéraires : enterré (un site) et exposé (un site) - et quelques sites villageois possibles situés soit au sommet de tertres artificiels (deux sites), soit sur des collines naturelles (seize sites).

Il est intéressant de souligner le nombre de "lieux spéciaux ", que ceux-ci présentent des monuments ou d'autres éléments non naturels : au total, il y a 31 sites de ce type. Ces sites ont une meilleure visibilité que les sites d'habitat, dont ils ne sont d'ailleurs pas toujours contemporains, et ils constituent un paysage riche en "lieux spéciaux». Plusieurs d'entre eux portent les traces de visites fréquentes et d'interventions multiples. Les fouilles que nous avons menées sur un site à structures mégalithiques ont montré exactement ce type d'acte, avec des caches votives évidentes et de multiples visites du monument.

\section{FouILLES DU SITE AP-CA-18}

Notre travail de fouille s'est concentré dans un site mégalithique spécifique, AP-CA-18, très bien préservé et avec des monolithes impressionnants dont certains de plus de $3 \mathrm{~m}$ au-dessus du sol (Figure 1). La surface fouillée atteint $71 \mathrm{~m}^{2}$, soit environ $15 \%$ de la surface totale du site. Afin d'interpréter la fonction des monuments, quelques questions fondamentales ont été retenues : comment le contenu du site est organisé, tant spatialement que chronologiquement ? Quels 


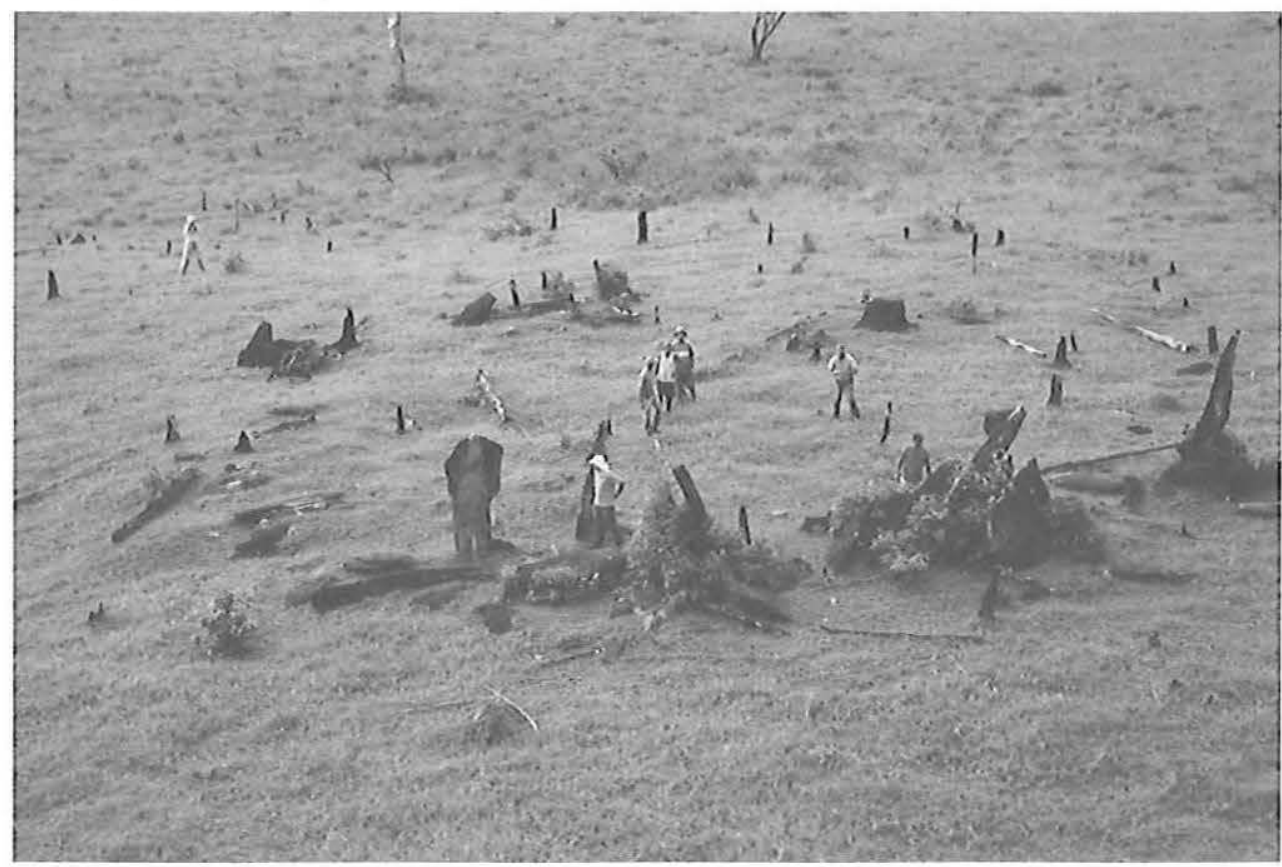

Fig. 1 - Vue générale de l'ensemble mégalithique principal du site AP-CA-18.

processus ont mené à l'agencement des monuments ? Par ailleurs, il nous fallait vérifier les processus taphonomiques ayant affecté les monolithes pour envisager des interprétations quant à la direction et à l'alignement de blocs de pierre particuliers.

Ce qui a été fouillé a été choisi en fonction des données de la prospection géophysique et des observations de terrain (Figure 2). Du côté de ces dernières, ce qui nous avait d'abord guidé, c'était l'implantation des blocs de pierre ; au fur et à mesure que les fouilles avançaient, les données obtenues sur les aires ouvertes ont modifié nos perspectives.

Au cours des fouilles, deux fosses funéraires ont été découvertes. La première, nommée par la suite " fosse 1 », a été trouvée à $20 \mathrm{~cm}$ sous la surface, à la limite nord de la fouille. Elle était associée à un grand dépôt de tessons et à un bloc granitique grossièrement circulaire. Bien que le bloc n'ait pas été exactement au-dessus de la fosse, nous l'avons tout de même interprété comme un couvercle, retiré par les occupants du site lors d'une nouvelle visite. Après avoir délimité le dépôt céramique et dégagé le bloc de granite (Figure 3a), le bloc et les tessons ont été enlevés, révélant une fosse circulaire taillée dans le niveau de la latérite et mesurant $160 \mathrm{~cm}$ de diamètre. Nous avons poursuivi la fouille et une grande 


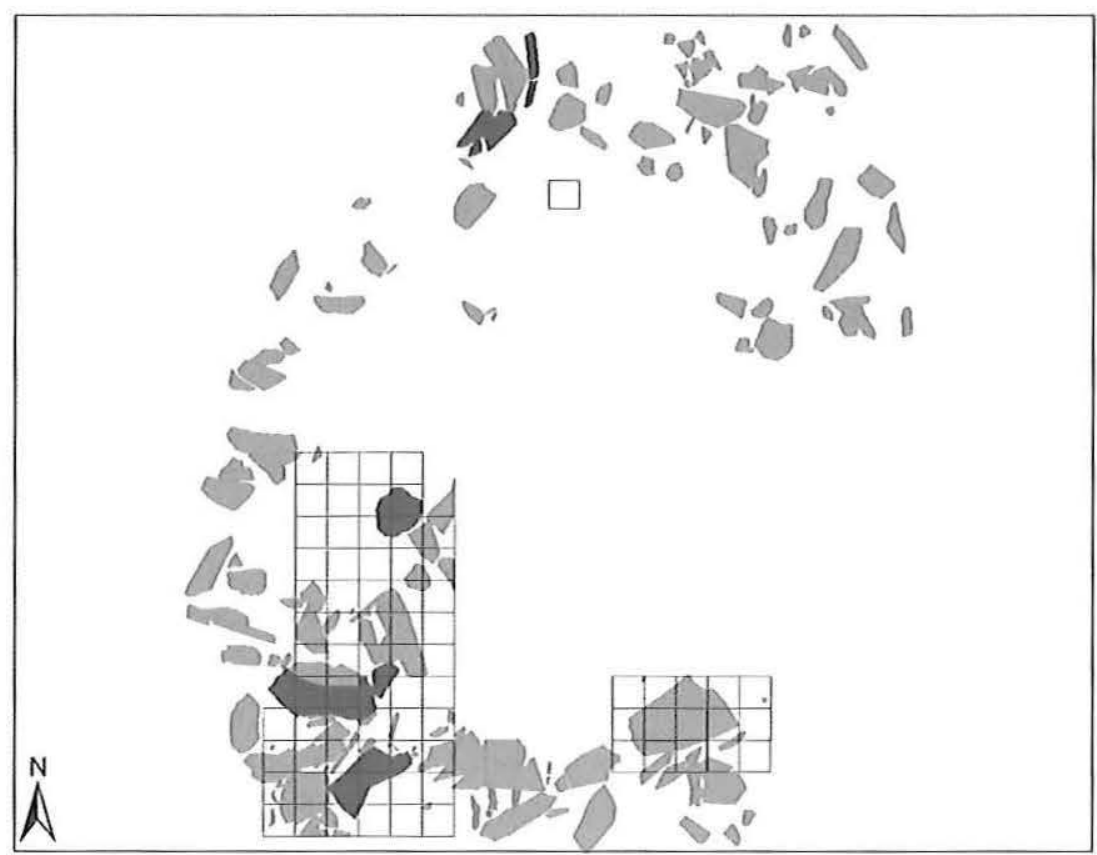

FIG. 2 - Localisation du quadrillage de fouille sur l'ensemble mégalithique principal de AP-CA-18 (blocs de pierre en gris ; le ton foncé pour les blocs verticaux, le clair pour les blocs couchés).
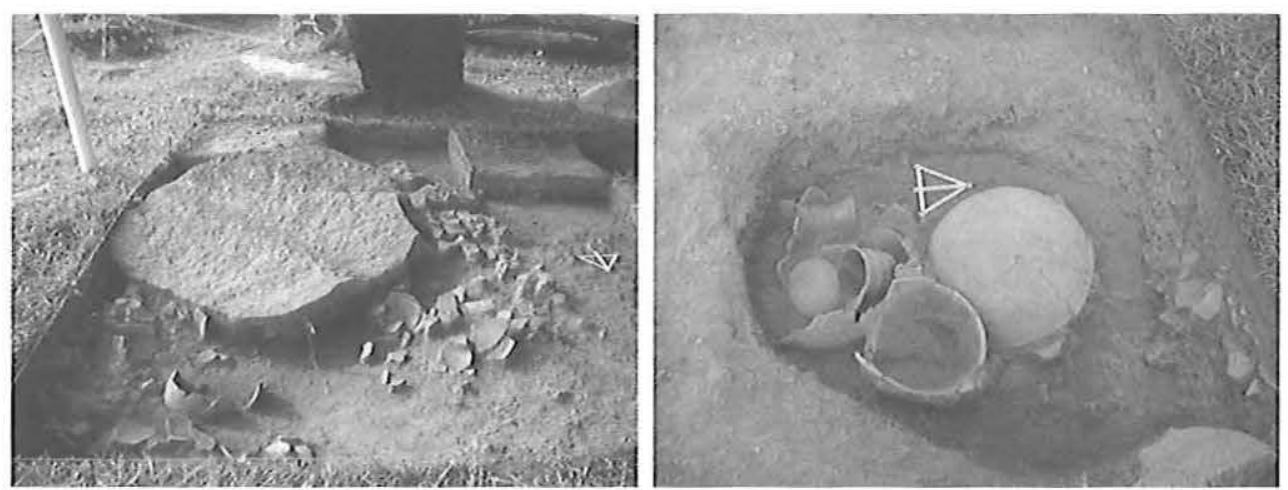

FIG. 3 - a) Le bloc de granite et le grand dépôt céramique qui ont recouvert et bordé la fosse 1 ;

b) À $50 \mathrm{~cm}$ de profondeur, la fosse devient évidente.

densité de tessons est encore apparue dans la continuité du dépôt autour du bloc de granite. La fosse est devenue encore plus évidente dans les niveaux inférieurs : elle est de forme circulaire et a été remplie de grands tessons et de récipients 
complets, écrasés les uns sur les autres (Figure 3b), ce qui suggère qu'ils ont été déposés dans la fosse avec peu de soin. À $40 \mathrm{~cm}$ de profondeur, il a été possible de distinguer de petites concentrations d'ossements brûlés dans certains récipients, indiquant leur fonction funéraire. L'ensemble des récipients presque entiers a continué jusqu'à $60 \mathrm{~cm}$ sous la surface.

De 60 à $120 \mathrm{~cm}$, la fosse était remplie de sédiment et de tessons de céramique. En dépit de la présence de quelques grands tessons, aucun autre récipient entier n'a pu être observé. En revanche, vers $130 \mathrm{~cm}$ de profondeur, des vases entiers ou presque, associés à de grandes quantités de charbon de bois, ont été à nouveau découverts. À $150 \mathrm{~cm}$, une chambre latérale a été repérée, orientée vers le nord et l'est. Trois récipients entièrement peints en rouge y avaient été délicatement déposés. La différence claire dans l'arrangement de ces vases par rapport à ce qui avait été vu plus haut suggère qu'à ce niveau profond un rituel plus complexe qu'un simple remplissage de fosse a eu lieu. Très peu de matériel a été trouvé dans les niveaux du fond du puits (de 180 à $190 \mathrm{~cm}$ ). À $190 \mathrm{~cm}$, la couche latéritique originale dans laquelle la fosse a été creusée a été atteinte. Le fait que trois vases complets aient été soigneusement placés à $20 \mathrm{~cm}$ au-dessus de la base de la fosse paraît indiquer qu'ils n'ont pas été déposés juste après le creusement de celle-ci. Notre hypothèse est qu'il y aurait eu une réutilisation du puits (Figure 4).
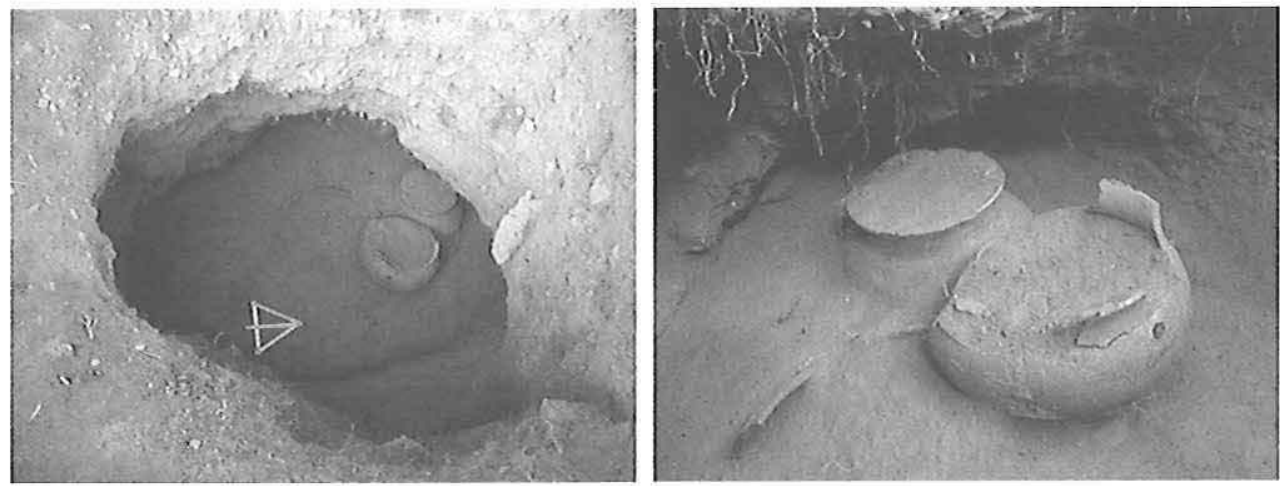

FIG. 4 - Vases déposés dans la chambre latérale de la fosse 1.

La seconde fosse funéraire a été trouvée dans la partie est de l'aire de fouille. Elle était localisée sous un large bloc de granite, mesurant $3,4 \mathrm{~m}$ par 2,6 m, disposé horizontalement. En fait, c'est le plus grand bloc du site. Par comparaison avec la fosse 1 , nous avons supposé que cette pierre avait été utilisée comme couvercle d'une autre fosse funéraire, ce qui a été effectivement confirmé. Les fouilles ont été étendues sur une superficie de $12 \mathrm{~m}^{2}$.

Dès le début de la fouille, nous avons noté que le dépôt était ici complètement différent de celui observé aux alentours de la fosse 1 : au lieu de grandes quantités 
de tessons et de vases brisés, seuls quelques rares petits tessons étaient présents. Après avoir dégagé le bloc mégalithique sur $10 \mathrm{~cm}$ de profondeur, celui-ci a pu être retiré, ce qui a permis de poursuivre la fouille.

Sous le couvercle, nous avons pu observer un arrangement grossièrement circulaire, constitué de petits blocs de granite. Ceux-ci étaient localisés exactement au sommet de l'ouverture de la fosse (Figure 5). Après avoir enlevé ces blocs atteignant jusqu'à $50 \mathrm{~cm}$ sous la surface, il a été possible de délimiter l'ouverture circulaire de la fosse. De 50 à $80 \mathrm{~cm}$, onze vases entiers ont été découverts, soigneusement disposés dans la fosse. Aucune chambre latérale, en revanche, n'a été détectée.

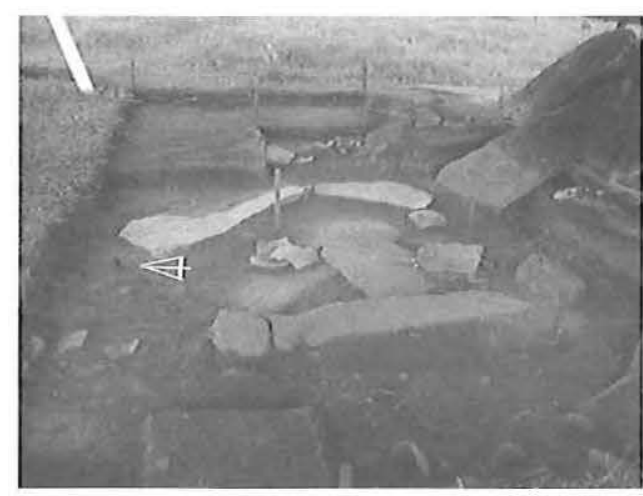

FIG. 5 - a) Blocs au sommet de la fosse 2 ;

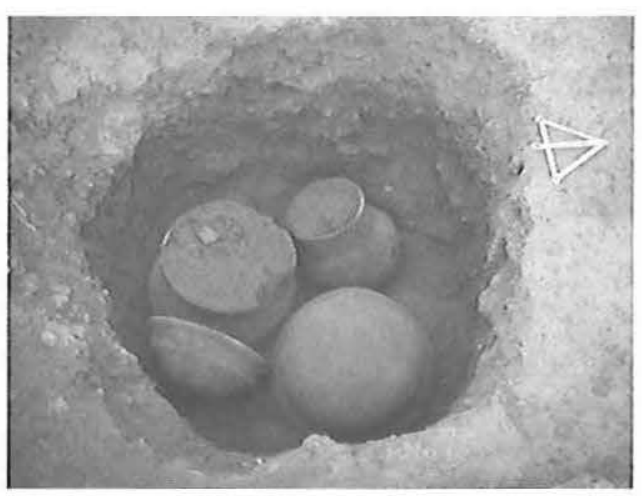

b) Récipients dans le fond de la fosse 2 .

Il est évident que les fosses 1 et 2 sont bien différentes. Dans la 2, les récipients étaient tous soigneusement déposés, selon un arrangement complexe incluant treize vases (onze complets et deux brisés). Parmi ces derniers, deux au moins sont funéraires (les plus grands du sommet de la fosse) : l'un contenait des ossements brûlés et l'autre des os non brûlés. Au regard de ces données, il est clair que la fosse 2 n'a jamais été ré-ouverte par ses constructeurs ou leurs descendants.

Plusieurs différences entre ces fosses méritent d'être notées et il ne s'agit pas seulement de forme ou de contenu. Bien que les deux aient eu une fonction funéraire, les activités pratiquées autour de l'une et de l'autre paraissent avoir été distinctes. Ce qui a été découvert dans la fosse 2 laisse penser qu'elle est le résultat d'un seul événement funéraire ; la fosse 1 en revanche semble avoir été ré-ouverte et réutilisée de manière fort différente. Les poteries n'y ont pas été délicatement déposées et les vases utilisés n'étaient pas entiers. À l'époque de la (ou des) réouverture(s) de la fosse 1, d'autres types d'activités ont dû être réalisés. Les distinctions observées laissent supposer par ailleurs une certaines variabilité des pratiques mortuaires.

Sur l'aire fouillée, il a été possible de délimiter plusieurs concentrations de tessons de céramique, indices de dépôts à ces endroits. Certaines étaient très 
importantes, s'étalant sur plus de $2 \mathrm{~m}$ de largeur et $30 \mathrm{~cm}$ d'épaisseur. D'autres étaient très petites et restreintes, et pourraient avoir été enveloppées dans des contenants organiques aujourd'hui disparus. Bien qu'aucun remontage n'ait encore été réalisé, les observations de terrain permettent d'affirmer que ces dépôts étaient constitués de nombreux tessons de céramiques diverses, et non de récipients entiers brisés. Nous pensons que ces contenants organiques auraient été remplis de tessons apportés d'ailleurs pour être déposés là, peut-être comme offrandes.

Un autre type de trouvaille concerne des dépôts de vases entiers. Pendant la fouille, nous avons pu constater que certains récipients avaient été placés dans des petits puits très étroits creusés dans le niveau latéritique. Les parois de ces fosses avaient été tapissées de tessons avant de déposer le vase. Aucune évidence macroscopique n'a été constatée sur ces vases; ils paraissent avoir été une sorte d'offrande.

Sur l'ensemble de l'aire de fouille, nous avons localisé deux endroits où des vases avaient été brisés in situ. Dans les deux cas, ces céramiques étaient directement associées avec des pierres dressées. Dans l'un des deux, une grande concentration de tessons remplissait trois vases brisés juste à la base d'un monolithe. Les tessons en question étaient fortement érodés, ce qui contrastait avec ceux des alentours et suggérait une exposition prolongée à l'air libre ; nous pensons que les céramiques-contenants ont été déposées entières et se sont brisées avec le temps. Dans le second cas, les vases étaient placés au milieu d'une enceinte de pierres dressées. Mais, ici, les vases ont été cassés au moment de leur dépôt, ce qui représente probablement un rituel de " mise à mort » des céramiques. Dans les deux cas, le caractère votif semble clair.

Un des principaux problèmes posé par le site est celui du pourquoi de l'érection éventuelle des blocs en position verticale et/ou oblique. C'est là un aspect essentiel pour tenter de comprendre comment les anciens groupes indigènes ont réellement disposé les monuments. Nous nous sommes donc particulièrement intéressés aux déplacements éventuels subis par les pierres dressées. Des fouilles contrôlées devaient permettre de dégager les sols pour reconnaître si des déplacements avaient eu lieu ou non pour établir les positions originelles des pierres, au cas où elles auraient été transplantées.

Comme la question des orientations astronomiques des monolithes a été soulevée durant le travail de terrain, ce qui implique des positionnements très spécifiques dans le sol, des recherches ont été entreprises sur le sujet et sur les aménagements à la base des monuments. Il était essentiel de ne provoquer aucun déplacement des pierres, afin d'offrir aux visiteurs et de préserver aux futurs chercheurs les mêmes conditions qu'aujourd'hui. Pour ne rien perturber, il a été choisi de n'étudier que des monolithes tombés avec l'aménagement de leur base intact.

La fouille a montré un système très élaboré de mise en place de chaque monolithe. Un puits elliptique a d'abord été creusé dans le niveau latéritique, 
légèrement plus grand que le bloc. L'insertion du bloc dans le puits est toujours soigneuse ; les plus grands espaces entre les parois du puits et le bloc ne dépassent pas $15 \mathrm{~cm}$ de largeur. Dans ces espaces, de petits blocs de granite et de latérite, de véritables coins de calage ont été intégrés, et l'ajustement de l'orientation du bloc a alors été fait. Il faut remarquer que les coins sur les côtés inclinés sont parfaitement collés au monolithe, ne laissant aucun champ pour bouger. De l'autre côté, d'autres coins scellaient les espaces vides et du sédiment servait également à les compacter. Ces arrangements soigneux des bases des monolithes confirment l'importance de leur positionnement.

\section{ANALySE CÉRAMIQUe PRÉLIMINAIRE}

Les fouilles ont fourni une grande quantité de tessons et de récipients entiers, qui n'ont pas encore été complètement analysés. En dépit de cela, quelques caractéristiques générales peuvent être dégagées afin de définir les principales tendances stylistiques et technologiques des céramiques trouvées.

Le dégraissant de la poterie récoltée est essentiellement composé de chamotte et de quartz. Dans certains récipients, des cailloux de granite broyés ont été utilisés comme dégraissant, donnant une texture grossière à la céramique. De grandes quantités de tessons étaient décorées par applications, incisions, ponctuations et peinture rouge (Figure 6), mais la majorité était sans décor. Très peu de peinture polychrome a été détectée.

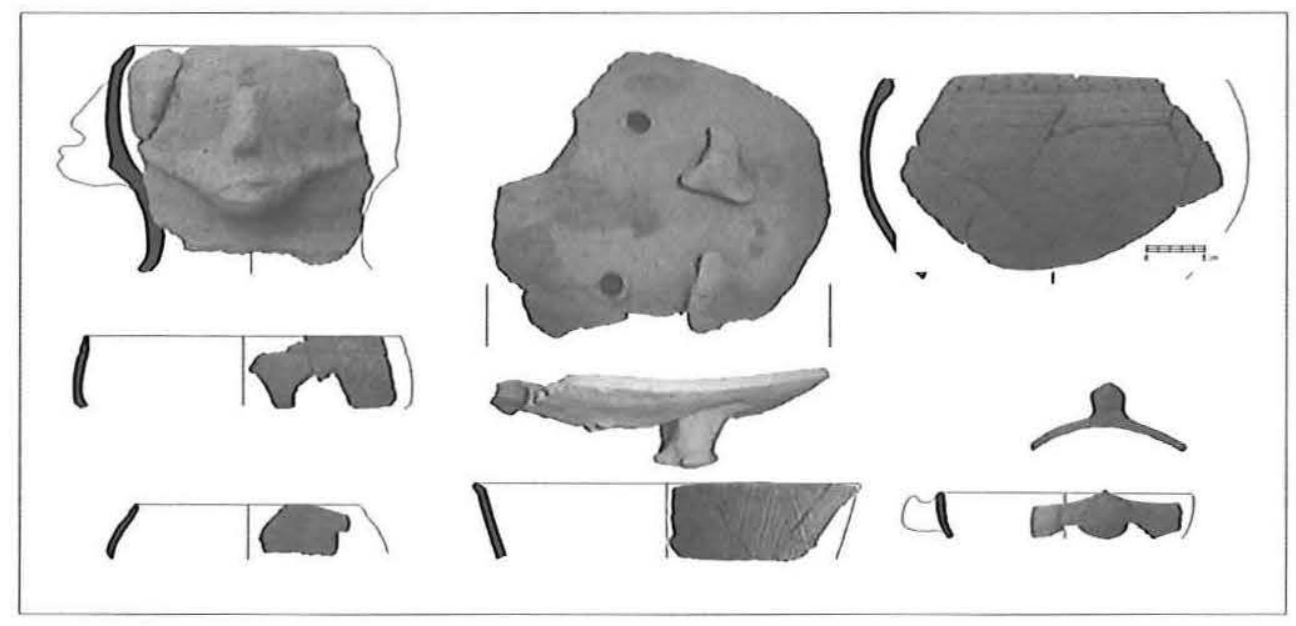

FIG. 6 - Motifs anthropomorphes et zoomorphes, peinture rouge et incisions. 
Les appliqués correspondent à des représentations zoomorphes modelées : oiseaux, mammifères, reptiles et amphibiens. Des représentations d'hommes ou de singes ont également été repérées. Le modelage était également utilisé pour former des visages humains sur l'encolure de certains vases.

Les incisions sont principalement associées à des ponctuations et, parfois, à de la peinture rouge. Les motifs des incisons sont doubles, parallèles, droits ou curvilignes. Ce type de décor connaît une grande distribution le long de l'Amazone et de l'Orénoque, et dans l'aire Caraïbe, et a été classé dans la tradition de « l'incision de lignes parallèles » (Zucchi 1991).

Étant donné ses caractéristiques technologiques, morphologiques et stylistiques, la céramique trouvée dans le site mégalithique AP-CA-18 doit être clairement associée à la phase Aristé (Meggers et Evans 1957) et, plus particulièrement, à son composant ancien daté au radiocarbone en Guyane française entre le $\mathrm{IV}^{\mathrm{c}}$ et le $\mathrm{x}^{\mathrm{e}}$ siècles apr. J.-C. (Rostain 1994).

\section{QUELQUES RELATIONS ENTRE LES BLOCS MÉGALITHIQUES ET L'ASTRONOMIE}

Des observations concernant le principal ensemble mégalithique d'APCA-18 ont été faites lors des solstices d'hiver durant trois ans, en 2005, 2006 et 2007. D'autres observations ont également été réalisées à l'équinoxe et au solstice d'été, mais aucune corrélation avec les blocs n'a pu être observée alors. Les données recueillies suggèrent des interprétations possibles sur l'utilisation du site comme marqueur temporel ou observatoire astronomique (Figure 7).

Deux corrélations au moins ont pu être supposées entre la disposition des pierres et le solstice de décembre. La première a été proposée par Elias José Ávila (IEPA, communication personnelle) et a été observée dès 2005. La position d'un bloc, légèrement incliné vers le sud, est telle que, durant l'après-midi du solstice (de 14h au crépuscule), les faces nord et sud du monolithe sont illuminées en même temps et ne montrent pas d'ombre (Figure 7).

Les observations menées durant les après-midi complets du solstice de décembre en 2006 et 2007 ont également permis une perception plus précise de l'inclinaison du bloc en relation (peut-être) avec la trajectoire du soleil (Figure 8). Cet alignement entre le soleil et le bloc paraît confirmer l'idée que les pierres étaient soigneusement dressées selon une observation astronomique et non de façon fortuite. Cela indique aussi que ces sites sont relativement bien conservés par rapport à leur état d'origine (Meggers et Evans 1957 ; Neves 2004).

Une seconde corrélation a pu être observée à l'aube du solstice de décembre 2006. Comme la fouille a eu lieu d'août à décembre, il a été possible de suivre les mouvements solaires sur une longue période. Des observations empiriques effectuées permettent de penser qu'un autre bloc (avec un trou) pourrait avoir été disposé face au lever du soleil. Cependant, à l'aurore, un autre bloc situé à 


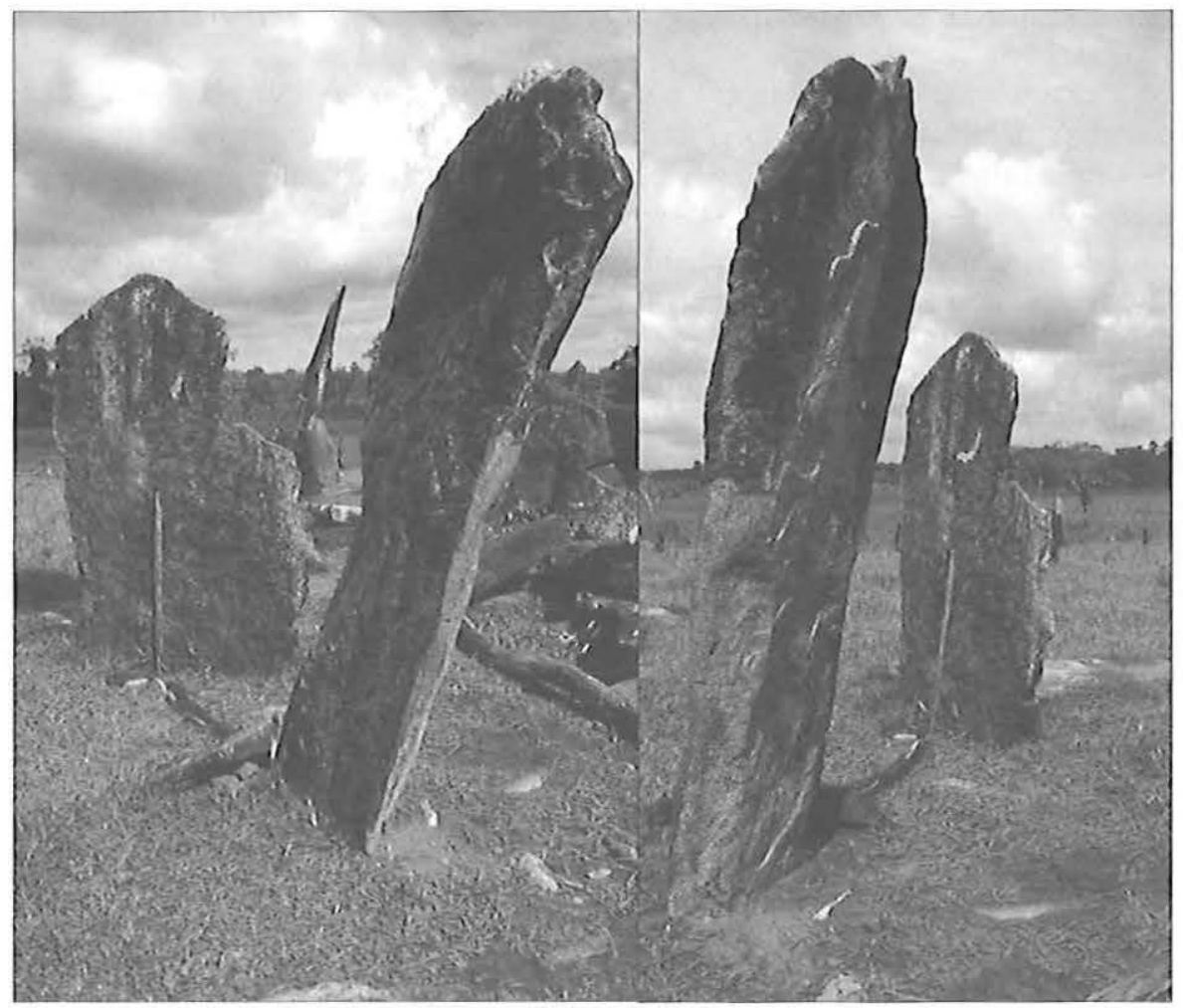

FIG. 7 - Durant le solstice de décembre 2005 (21/12/2005), à 14h30, les faces nord (à gauche) et sud du bloc du premier plan n'ont pas d'ombre. La seule ombre projetée est vers l'est : elle est fine comme le bloc lui-même.

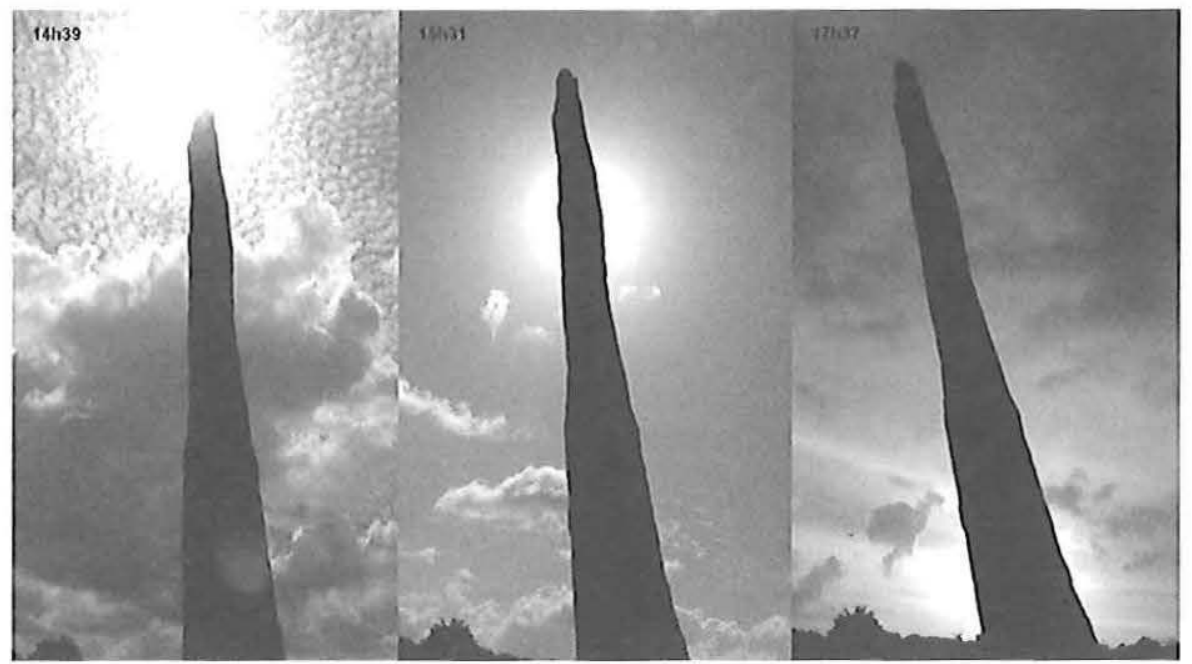

FIG. 8 - Vue du soleil juste au-dessus et à l'arrière du bloc précédent, durant l'après-midi du solstice (21/12/2006), montrant leur alignement. 
l'arrière de celui observé, bloquait la vue. Mais lorsque le soleil apparut enfin, il se situait juste à l'endroit où les plus hautes parties des deux blocs étaient alignées (Figure 9c).

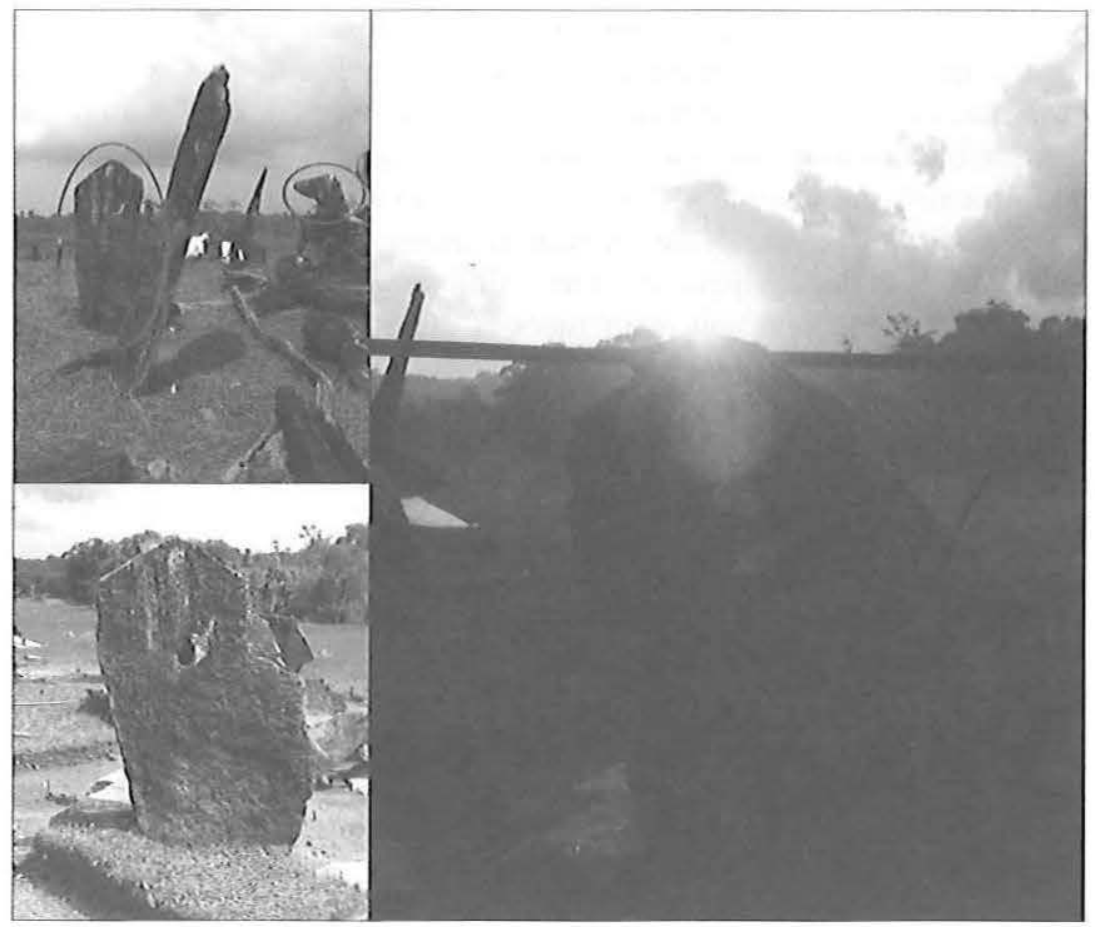

FIG. 9 - a) Blocs de pierre alignés peut-être en fonction de l'aube du solstice ;

b) Alignement des pierres durant la journée ;

c) Même alignement à l'aube du solstice (21/12/2006, 6h50).

Il est probable que des savoirs astronomiques existaient chez de nombreux groupes amérindiens (Green et Green 2006; Campos 2006). Les anciennes populations qui avaient ces connaissances auraient très bien pu avoir matérialisé ces savoirs à travers quelque chose de concret et durable. Ce constat ouvre de nouvelles perspectives pour l'étude de l'astronomie chez les anciennes populations locales, pour lesquelles le ciel servait, sans doute, non seulement comme un marqueur temporel, mais aussi comme un marqueur spatial (à titre comparatif, on peut s'appuyer sur les groupes palikur actuels qui vivent un peu plus au nord : Green et Green 2003). Le paysage construit par les monolithes aurait ainsi une relation avec l'espace stellaire. 


\section{Conclusion}

La recherche entreprise sur le sujet n'est pas achevée, mais quelques remarques de conclusion peuvent, d'ores et déjà, être proposées. Les fouilles ont démontré que le site mégalithique AP-CA-18, tel que l'on peut le voir aujourd'hui, est en fait un palimpseste de différents types de dépôts à l'intérieur d'un ensemble mégalithique. La chronologie précise de chacun des événements est pratiquement impossible à faire, mais ceux-ci peuvent être classés comme suit : épisodes d'ouverture de fosses pour arranger et fixer les monolithes selon des inclinaisons et des alignements précis ; différentes sortes d'offrandes céramiques autour des blocs, rituellement tuées et dépôt de tessons à l'intérieur de récipients non conservés, placement de poteries entières à la base des blocs ; ouverture de puits étroits pour déposer des vases entiers ; creusement de fosses de différentes tailles pour des dépôts funéraires et fermeture avec un couvercle de pierre horizontal ; réouverture de certaines d'entre elles et déplacement du matériel contenu avec ajout de nouveau matériel.

Plusieurs questions restent sans réponse et devraient être approfondies grâce à de nouvelles analyses, d'autres collectes de données et de futures interventions dans d'autres sites de la région. Au niveau du site, la chronologie d'érection des mégalithes devrait être précisée, cela afin de comprendre notamment si les mégalithes ont été disposées sur une période courte ou s'ils résultent de l'agrégation de différents éléments sur une longue période de temps. La connaissance de la durée de la construction est essentielle pour saisir le degré d'implication des sociétés dans l'érection des mégalithes.

L'étude de la chronologie et l'analyse des sépultures s'imposent également. Une des questions soulevée par les urnes funéraires concerne évidemment le type de relation qui a pu exister ou non entre les individus enterrés.

D'autres questions ne pourraient être posées et éventuellement résolues qu'après que des prospections et des fouilles extensives aient été réalisées dans d'autres sites de la région. Il faut en particulier préciser les liens entre les sites mégalithiques, comme lieux de dépôts funéraires et de rituels, et les autres types de sites funéraires (grottes et cimetières de plein air). Par ailleurs la question des relations avec les villages demeure entière puisque très peu ont été détectés, ce qui contraste fortement avec l'impressionnante quantité de sites spéciaux et votifs. Enfin notre recherche doit s'orienter vers la datation plus systématique d'un grand nombre de sites possibles, afin de disposer d'une trame générale sur l'occupation régionale où l'on situera ainsi mieux l'émergence et le développement des sites à mégalithes. *

\footnotetext{
* Manuscrit reçu en juin 2008, accepté pour publication en septembre 2008.
} 


\section{NoTES}

Remerciements : nous voudrions grandement remercier Stéphen Rostain pour ses encouragements à la publication de cet article et pour l'avoir aimablement traduit de l'anglais. Nous remercions également notre institut pour tout son appui, ainsi que les nombreux participants à toutes les étapes du travail, qui ont rendu ce projet possible.

1. Initiales de la région (Amapá), de la commune (Calçoene) et du numéro de site (18).

2. Ce site sub-aquatique fut enregistré comme une partie possible d'un site villageois proche, localisé sur une colline naturelle. En tant que tel, dans le compte final il est présenté parmi les 16 sites de villages possibles sur colline naturelle.

\section{RÉFÉRENCES}

\section{Campos Marcio D.}

2006 "A cosmologia dos Caiapó », Scientific American Brasil, edição especial 14, pp. 62-71.

\section{Ferreira Penna Domingo Soares}

1879 «Urnas do Maracá », Archivos do Museu Nacional, 2, pp. 69-71.

\section{Goeldi Emilio}

1905 Excavações Arqueológical em 1895. 1 parte : as cavernas funerarias atificiaes dos indios hoje extinctos no rio Cunany (Goanany) e sua ceramica, Memórias do Museu Paraense de Historia Natural e Ethnographia, 1, Belém.

\section{Green Lesley F. et David R. Green}

2003 "From chronological to spatio-temporal histories: mapping heritage in Arukwa, Área Indígena do Uaçá, Brazil », History and Anthropology, (14) 3, pp. 1-13.

2006 Kayeb : a constelação anaconda bicéfala dos Palikur, Cape Town, pp. 1-8 [manuscrit].

\section{Meggers Betty J. et Clifford Evans}

1957 Archaeological imvestigations at the mouth of the Amazon, US Government print office, Smithsonian Institute, coll. « Bulletin of the Bureau of American Ethnology » 167, Washington.

Neves Eduardo G.

2004 "Introduction: the relevance of Curt Nimuendajú's Archaeological Work ", in Curt Nimuendajú, In Pursuit of a Past Amazon. Archaeological researches in the Brazilian Guyana and in the Amazon region by Curt Nimuendajú, a posthumous work compiled and translated by Stig Rydén and Per Stenborg, Världskulturmuseet, coll. « Ethnological Studies » 45, Göteborg, pp. $2-8$. 


\section{Nimuendajú Curt}

2004 In Pursuit of a Past Amazon. Archaeological researches in the Brazilian Guyana and in the Amazon region by Curt Nimuendaju, a posthumous work compiled and translated by Stig Rydén and Per Stenborg, Världskulturmuseet, coll. « Ethnological Studies » 45, Göteborg.

\section{Rostain Stéphen}

1994 L'occupation amérindienne ancienne du littoral de Guyane, Éditions de l'ORSTOM, coll. « Travaux et Documents Micro-fichés » 129, Paris.

\section{Zucchi Alberta}

1991 "Prehispanic connections between the Orinoco, the Amazon, and the Caribbean Area ", Proceedings of the Thirteenth International Congress for Caribbean Archaeology, Curaçao, Netherlands Antilles, pp. 202-220. 